\title{
Implementation of a ward round pro-forma to improve adherence to best practice guidelines
}

\author{
Xavier Boland \\ Royal Cornwall Hospital NHS Trust, Cornwall, United Kingdom
}

\begin{abstract}
Our aim was firstly to assess adherence to best practice guidelines for the prevention of healthcare associated causes of inpatient mortality and morbidity by junior doctors. Secondly, we wanted to measure the impact of a ward round checklist on rates of adherence.
\end{abstract}

The rates of correct prescribing of antibiotics, venous thrombo-embolism prophylaxis, and oxygen (pro re nata) as well as correctly completed paperwork for peripheral venous cannulas were measured in a spot audit of all medical notes of patients on a medical assessment unit. This was repeated two weeks and two months after the introduction of a specifically designed ward round checklist for junior doctors.

Initial audit of 40 patient notes confirmed generally poor compliance with best practice guidelines in the prescription of antibiotics (58\% correctly prescribed) and oxygen (42\%), and in the use cannula care plans (39\%). Venous thrombo-embolism prophylaxis prescribing on the other hand was widespread (82\%). The introduction and extensive use of the ward round checklist did not have a significant impact on these figures as shown in the two following stop audits ( 30 and 36 notes respectively).

Checklists are helpful in providing a structured and systematic approach to complex tasks and have been shown to have a measurable impact in improving patient care. Their effectiveness is however limited by their uptake and regular correct use. Obstructing issues include poor understanding of the need for change in practice, lack of individual accountability and variable involvement of clinical leaders. These issues must be addressed together in order to effect a successful change in clinical practice.

\section{Problem}

Health care associated infections, hospital acquired venous thromboembolism (VTE), and oxygen induced hypercapnia are largely preventable causes of morbidity and mortality in hospital inpatient populations.[1-3] Targeted evidence-based guidelines have been designed to provide a structured approach for their prevention and, like other evidence-based clinical guidelines, have been shown to have a significant impact in improving patient outcomes.[4] Poor adherence to evidence-based guidelines by clinical staff however can reduce their impact and allow preventable problems to persist. Likewise, without a strategy for their implementation, clinical guidelines are generally ineffective in changing the behaviour of health care professionals.[5]

This study has two main aims. Firstly, to measure the adherence to specific best practice guidelines for the prevention hospital acquired causes of mortality and morbidity (HACMMs) by doctors on a medical assessment unit in regional teaching hospital. Secondly, to measure the effectiveness of the study's interventional checklist (the ward round pro-forma) on rates of adherence to best practice guidelines.

\section{Background}

Interventions directed at preventing health care associated causes of in-hospital morbidity and mortality (HACMMs) include correctly prescribing specific drugs whose overuse or omission could potentially harm the patient and ensuring, through the use of targeted documentation, that peripheral venous cannulas are correctly managed throughout a patient's hospital stay.

This study focuses on the following recommendations for prevention of HACMMs:

1. Oxygen should be prescribed to achieve target saturations of $94-98 \%$ for most acutely ill patients or $88-92 \%$ for those at risk of hypercapnic failure. British Thoracic Society guidelines [6]

2. All patients admitted to hospital and assessed as high risk of venous thromboembolism should receive correct anticoagulation. NICE Guidelines [7]

3. All prescriptions of antibiotics must have an indication and a stop/review date listed on the drug chart. Local policy

4. Care plans should be used for peripherally inserted cannulas as reminders to review their continuing use or prompt their removal. Local Policy and Healthcare Infection Society Guidelines.[8]

Many of the above interventions need to be initiated early on in the patient's journey through hospital. When reviewing patients on a post-take ward round (ie shortly after they have been admitted), the medical team has an opportunity to ensure these issues have been addressed and correct actions taken. However, the ward round is often a complex process and it is easy for parts of it to be forgotten 
BMJ Quality Improvement Reports

or missed out. Checklists can be helpful in reducing errors and omissions when performing complex tasks.[9] The WHO surgical safety checklist for example has been shown to have a significant impact in reducing rates of surgical complications [10] while also improving communication between health professionals. This study tests the effectiveness of similar checklist designed specifically for medical ward round in improving compliance to clinical guidelines.

\section{Baseline measurement}

A spot audit was conducted on the medical assessment unit (MAU) of a regional teaching hospital. The medical notes, nursing notes, and drug chart of all the patients on the unit at the time of data collection were included in the study. Of these patients, the following were excluded: those who had not yet been reviewed by a doctor or been seen on a post-take ward round, and those whose notes were incomplete or unavailable at the time. Data were collected using a standardised data collection form. The patients' working diagnoses and background medical histories were recorded from the medical clerking. The drug charts were checked to see if patients were prescribed oxygen (pro re nata), deep vein thrombosis (DVT) prophylaxis, and/or antibiotics. If a patient was on an antibiotic, the prescription was checked for an accompanying indication and a stop or review date. If a patient was not prescribed DVT prophylaxis, notes were checked for evidence of a completed venous thromboembolism risk assessment form. For patients with a peripheral venous cannula, the nursing notes were checked for a completed cannula care plan. Data collection took place after the end of the junior doctor day shift (5pm) and doctors were not told that the audit was taking place.

Forty patients were included in the initial spot audit with a median age of 48 . Of these, 32 (89\%) were prescribed appropriate DVT prophylaxis or had the correct paperwork filled out when not. Seventeen out of $40(42 \%)$ were prescribed oxygen and of the 24 patients who were prescribed an antibiotic, $14(58 \%)$ had an indication and stop/review date included in the charting. Twentyeight patients had a cannula of which 11 (39\%) had a cannula care plan. Of the 21 patients with either a background history of cardiac or respiratory disease or a working diagnosis involving the cardiac or respiratory systems, 13 (62\%) were prescribed oxygen. Overall, the initial audit confirmed poor compliance to best practice guidelines for the prevention of HACMMs except in the prescription of DVT prophylaxis. This was in keeping with previous audits run on the unit in the previous 12 months.

See supplementary file: ds4606.pdf - "APPENDIX 1"

\section{Design}

A specifically designed ward round pro-forma (WRP) was introduced on the unit and made available to all junior doctors involved in ward rounds. The ward round pro-forma [appendix 1] was designed as a jobs list with a series of prompts for doctors to check that the following had been completed or discussed and considered:
1. Oxygen prescribing

2. Venous thromboembolism prophylaxis prescribing

3. Antibiotic prescribing with indication and stop or review date, and

4. Completion of a cannula care plan.

(Also included were prompts to check for completion of dementia screen, decision on the patient's resuscitation status, and review of proton pump inhibitor prescription, although these were not included in this study). Several medical consultants working in MAU were involved in designing the checklist and deciding which aspects of patient care should be included. Junior doctors were informed of the introduction of the WRP during morning hand over meetings and in emails explaining its use. Copies of the WRP were stored openly on the unit where they were freely available for daily use.

\section{Strategy}

Adherence to guidelines was audited twice further at two weeks and two months following the introduction of the WRP. In the time between the second and third spot audits, a meeting was arranged with junior doctors to go over the rationale behind the project and reiterate the recommendations of the relevant guidelines. Junior doctors were also interviewed regularly to identify potential issues and collect feedback.

\section{Results}

The research team noted good uptake of the ward round pro-forma by junior doctors. This was confirmed by clerical staff who were regularly asked by junior doctors to print further copies of the proforma when stocks were running low.

One of the main difficulties we found was in encouraging junior doctors to make regular use of the checklist as well as the job's list. Discussions with junior staff highlighted several obstacles to its routine use. For example:

- Staff often felt under pressure to see patients rapidly so felt they did not have the time to deal with all the items of the checklist, and as a result it was often left out altogether

\footnotetext{
- Many junior doctors were either unaware of the existence of the guidelines in question or thought that the ward was already compliant with them
}

- There was some confusion regarding the recommendations of the guidelines and their exact purpose. Some staff felt some of the clinical guidelines were simply there to meet hospital targets but were unclear as to their role in enhancing patient safety

- Often the task of checking whether the guidelines had been followed fell between staff member's perceived individual roles. As a result, tasks were left undone on the assumption that someone else would do them

- There was a general lack of accountability. For example, when an 
antibiotic was not prescribed correctly, the prescriber was not informed of the error. By that same token, there was rarely any feedback to reinforce positive behaviour when prescriptions were done correctly

- Awareness of the WRP was variable among senior staff including doctors external to the medical assessment unit who were involved in post take ward rounds. This sent a mixed message to junior staff.

The second and third spot audits included 30 and 36 patients respectively with median ages of 47.5 and 45 . These showed no significant changes in rates of prescription of oxygen or DVT prophylaxis, with the latter remaining elevated in both subsequent spot audits $(87 \%, 89 \%)$. Similarly, antibiotics continued to be prescribed incorrectly (without an indication and/or without a stop/review date) in about a third of cases and rates of oxygen prescribing for patients likely to require oxygen were largely unchanged. However, there was a marked improvement in the completion of cannula care plans from $39 \%$ to $80 \%$ and $68 \%$. The results of the all three audits were recorded in SPSS (IBM SPSS version 19.0) and the difference in rates of compliance to guidelines was analysed for statistical significance using a Pearson's Chi squared test. $P$ values greater than 0.05 were considered statistically significant. Results are summarised in table 1.

See supplementary file: ds4837.xlsx - "Table 1"

\section{Lessons and limitations}

The primary goal of our intervention was to introduce a new structured approach to the MAU ward round that specifically targeted the prevention of hospital acquired causes of morbidity and mortality (HACMMs). The checklist provided a simple, inexpensive, and unobtrusive tool for this. As it was coupled with a jobs list, it proved to be of practical value in the junior doctors' day to day work without creating additional paperwork.

However, despite its widespread use during ward rounds on MAU, it was found to have little measurable impact on the adherence to guidelines for the prevention of HACMMs. Whereas the study's intervention focused largely on changing individual doctors' practice, feedback from staff underlined the importance of the work environment on shaping behaviour. This emphasised the need to take into account the multitude of factors that influence individuals to change their practice when promoting change.

Omitting certain immutable factors beyond the scope of this study (eg, reducing the individual doctor's workload) recommendations of the study can be grouped around four main themes. These are broadly based on a Harvard Business School paper on leading change in corporate institutions [11] and mirror some of the opinions expressed in journal publications on the implementation of clinical guidelines [12-14]:

1. Accountability/ownership: Junior doctors and admitting doctors on the unit should be encouraged to take ownership of the problem of HACMMs. Junior doctors should be empowered to discuss these issues during ward rounds and should be given a role in finding solutions to the problem. Their individual responsibilities with regards to the implementation of specific guidelines should be decided on induction

2. Leadership: Efforts should focus on securing the involvement of clinical leaders (in this case medical consultants) in promoting change of practice. This would send a strong and a consistent message to junior staff about the importance of complying with best practice guidelines

3. Communication: The goals of achieving high rates of compliance to targeted guideline recommendations should be clearly stated along with the basis for the changes in practice. This message should be reinforced routinely not only through email conversations and ward meetings, but in day to day interaction on the ward. These aims should outlined during junior doctor's induction and regularly recapitulated throughout their time on MAU

4. Audit: Rates of adherence to best practice guidelines on the unit and, if possible, of individual doctors should be routinely audited. This would be a source of feedback for ward staff but would also provide benchmarks against which short term targets could be set. Similarly, ward round checklists could be reviewed by senior staff, nurses or pharmacist at the end of a shift to check whether they have been correctly used and significant issues addressed.

To effect successful change in practice across the unit, a coordinated effort would need to be made to tackle all four recommendations in concert. The aim would be to create an environment where junior doctors feel confident that changing their individual practice would be likely to have a positive and sustained impact on patient care.

Study Limitations: No control group was included in this study so we cannot tell if changes in the results are specific to the medical assessment unit (MAU) or are hospital wide. However, it is worth noting that because most patients admitted to medical wards come through the MAU, the rates of adherence to guidelines in MAU would be reflected across other wards. Lack of blinding in the collection of data exposed the chance of observer bias. Small sample size and restriction of spot auditing to single day shifts also allowed for greater variability between the results. A variety of factors could affect measured outcomes on a specific shift including the number of patients admitted to the unit in the day, the complexity of cases and number of staff on the unit during the shift. On a busy shift with reduced support or supervision from senior staff, a junior doctor is more likely to make an error or omission when prescribing for a patient.

\section{Conclusion}

Whereas checklists have been shown to be successful in providing a systematic approach to complex problems in a multitude of settings, their value is largely dependent on creating an environment where they are used routinely and in the correct way. To motivate and empower staff to change their practice multiple approaches likely need to be employed together including: 1 . Making sure staff are well informed regarding the need for change 


\section{BMJ Quality Improvement Reports}

1. Giving junior staff a sense of ownership and accountability with regards to the issues at hand 3 . Involving clinical leaders

2. Making culture change part of everyday discussions, and

3. Regularly auditing progress.

\section{References}

1. Bump GM, Dandu M, Kaufman S. How complete is the evidence for thromboembolism prophylaxis in general medicine patients? A meta-analysis of randomized controlled trials. J Hosp Med 2009 May;4(5):289-97.

2. Austin M, Wills K, Blizzard L. Effect of high flow oxygen on mortality in chronic obstructive pulmonary disease patients in pre-hospital setting: randomised controlled trial. BMJ 2010 Oct 18;341.

3. Valiquette L, Cossette B, Garant MP. Impact of a reduction in the use of high-risk antibiotics on the course of an epidemic of clostridium difficile-associated disease caused by the hypervirulent NAP1/027 strain. Clin Infect Dis 2007 Sept 1;45.

4. Grimshaw JM, Russell IT. Effect of clinical guidelines on medical practice: a systematic review of rigorous evaluations. Lancet 1993. 342:1317-22.

5. Freemantle N, Harvey E, Grimshaw JM. The effectiveness of printed educational materials in changing the behavior of health care professionals. In: Cochrane Collaboration. Cochrane Library. 1996. Issue 3. Oxford: Update Software.

6. O'Driscoll BR, Howard LS, Davidson AG. BTS guideline for emergency oxygen use in adult patients. Thorax 2008 Oct;63 Supplement 6.

7. Venous Thromboembolism: Reducing the Risk of Venous Thromboembolism (Deep Vein Thrombosis and Pulmonary Embolism) in Patients Admitted to Hospital. Royal College of Physicians. 2010. NICE Clinical Guidelines. No. 92

8. Loved HP, Wilson JA, Pratt RJ. Epic3: National EvidenceBased Guidelines for Preventing Healthcare-Associated Infections in NHS Hospitals in England. J Hosp Infect 2014 Jan;86. Supplement 1.

9. Herring R, Caldwell G. Implementation of a considerative checklist to improve productivity and team working on medical ward rounds. Clinical Governance: An International Journal. 2011. 16(2): 129-36.

10. Haynes AB, Weiser TG, Berry WR. A surgical safety checklist to reduce morbidity and mortality in a global population. NEJM 2009 Jan 29; 360(5): 491-9.

11. Kotter J. Leading Change: Why Transformation Efforts Fail." Harvard Business Review. 1995 March-April: 1-10.

12. Feder G, Eccles M, Grol R. Using clinical guidelines. BMJ 1999. 318:728-30.

13. Grimshaw JM, Russell IT. Achieving health gain through clinical guidelines II: Ensuring guidelines change medical practice. Quality in Health Care 1994;3:45-52

14. Williams JG, Cheung WY, Price DE. Clinical guidelines online: do they improve compliance? Postgrad Med J. 2004;80:415-9

\section{Declaration of interests}

Nothing to declare.

\section{Acknowledgements}

Joanne Palmer. 